\title{
FASN, ErbB2-mediated glycolysis is required for breast cancer cell migration
}

\author{
LAN ZHOU $^{1,2,4}$, SUFANG JIANG ${ }^{1}$, QIANG FU ${ }^{1}$, KELLY SMITH ${ }^{3}$, \\ KAILING TU ${ }^{1}, \mathrm{HUA} \mathrm{LI}^{2}$ and YUHUA ZHAO ${ }^{1}$ \\ Departments of ${ }^{1}$ Biochemistry and Molecular Biology, ${ }^{2}$ Anatomy, West China School of Preclinical \\ and Forensic Medicine, Sichuan University, Chengdu, Sichuan 610041, P.R. China; \\ ${ }^{3}$ Mitchell Cancer Institute, University of South Alabama, Mobile, AL 36604, USA
}

Received November 23, 2015; Accepted December 29, 2015

DOI: $10.3892 /$ or.2016.4627

\begin{abstract}
Both fatty acid synthase (FASN) and ErbB2 have been shown to promote breast cancer cell migration. However, the underlying molecular mechanism remains poorly understood and there is no reported evidence that directly links glycolysis to breast cancer cell migration. In this study, we investigated the role of FASN, ErbB2-mediated glycolysis in breast cancer cell migration. First, we compared lactate dehydrogenase A (LDHA) protein levels, glycolysis and cell migration between FASN, ErbB2-overexpressing SK-BR-3 cells and FASN, ErbB2-low-expressing MCF7 cells. Then, SK-BR-3 cells were treated with cerulenin (Cer), an inhibitor of FASN, and ErbB2, LDHA protein levels, glycolysis, and cell migration were detected. Next, we transiently transfected
\end{abstract}

Correspondence to: Dr Hua Li, Department of Anatomy, West China School of Preclinical and Forensic Medicine, Sichuan University, No. 17, Section 3, South Renmin Road, Chengdu, Sichuan 610041, P.R. China

E-mail: lihua_scu@scu.edu.cn

Dr Yuhua Zhao, Department of Biochemistry and Molecular Biology, West China School of Preclinical and Forensic Medicine, Sichuan University, No. 17, Section 3, South Renmin Road, Chengdu, Sichuan 610041, P.R. China

E-mail: zhaoyh@scu.edu.cn

Present address: ${ }^{4}$ School of Basic Medical Sciences and Nursing, Chengdu University, Chengdu, Sichuan 610015, P.R. China

Abbreviations: 2-DG, 2-deoxyglucose; Cer, cerulenin; EGF, epidermal growth factor; EMT, epithelial-mesenchymal transition; FASN, fatty acid synthase; FBS, fetal bovine serum; GAPDH, glyceraldehyde-3-phosphate dehydrogenase; HRG- $\beta 1$, heregulin- $\beta 1$; HRP, horseradish peroxidase; LDHA, lactate dehydrogenase A; MAPK, mitogen-activated protein kinase; OX, oxamate; PI3K, phosphatidylinositol 3 kinase; PS, penicillin and streptomycin; TBS, Tris buffered saline; TNFR, tumor necrosis factor receptor

Key words: fatty acid synthase, ErbB2, glycolysis, migration, breast cancer
ErbB2 plasmid into MCF7 cells and detected FASN, LDHA protein levels, glycolysis and cell migration. Heregulin- $\beta 1$ (HRG- $\beta 1$ ) is an activator of ErbB2 and 2-deoxyglucose (2-DG) and oxamate (OX) are inhibitors of glycolysis. MCF7 cells were treated with HRG- $\beta 1$ alone, HRG- $\beta 1$ plus $2-D G$, OX or cerulenin and glycolysis, and cell migration were measured. We found that FASN, ErbB2-high-expressing SK-BR-3 cells displayed higher levels of glycolysis and migration than FASN, ErbB2-low-expressing MCF7 cells. Inhibition of FASN by cerulenin impaired glycolysis and migration in SK-BR-3 cells. Transient overexpression of ErbB2 in MCF7 cells promotes glycolysis and migration. Moreover, 2-deoxyglucose (2-DG), oxamate (OX), or cerulenin partially reverses heregulin- $\beta 1$ (HRG- $\beta 1)$-induced glycolysis and migration in MCF7 cells. In conclusion, this study demonstrates that FASN, ErbB2-mediated glycolysis is required for breast cancer cell migration. These novel findings indicate that targeting FASN, ErbB2-mediated glycolysis may be a new approach to reverse breast cancer cell migration.

\section{Introduction}

Breast cancer is the most common type of cancer and remains the leading cause of cancer death among women in the world (1). Metastasis is the main cause of breast cancer death (2). Breast cancer metastasis is a complex pathophysiological process involving epithelial-mesenchymal transition (EMT), migration and motility, invasion, and metastatic growth (3). The molecular mechanism underlying breast cancer metastasis is not fully understood.

Mounting evidence shows that cancer cells are different from normal cells in their metabolic properties. Normal cells mostly depend on mitochondrial oxidative phosphorylation to produce energy in the form of ATP. In contrast, cancer cells prefer breakdown of glucose for energy rather than oxidative phosphorylation, even in the presence of available oxygen. This phenomenon is known as aerobic glycolysis, or Warburg effect, which is a hallmark of cancer cells (4-9). However, there is no report that directly links increased glycolysis to breast cancer cell migration.

Fatty acid synthase (FASN) is a key enzyme involved in neoplastic lipogenesis and the production of long-chain fatty 
acids from acetyl-coenzyme A (CoA) and malonyl-CoA. In breast cancer cells, there is a positive correlation between overexpression of FASN and amplification and/or overexpression of ErbB2 (10,11). ErbB2 (Her2/neu) is an oncogene that is overexpressed in $25-30 \%$ of human primary breast cancer and is associated with a poor prognosis (12). It has been demonstrated that both ErbB2 and FASN enhance the transformation and/or metastatic potentials of breast cancer cells (13-18). Our previous study showed that ErbB2 promotes breast cancer cell glycolysis via upregulation of lactate dehydrogenase A (LDHA), a key enzyme that catalyzes the production of lactate (19). Therefore, we hypothesize that both FASN and ErbB2 promote cell migration via glycolysis in breast cancer.

Heregulin- $\beta 1$ (HRG- $\beta 1$, also known as neuregulin- $\beta 1)$, a member of the epidermal growth factor (EGF) family (20), is an ErbB3 and ErbB4 ligand that leads to the formation of ErbB2-ErbB3/ErbB4 heterodimers, resulting in activation of the ErbB2 signaling pathway (21). HRG- $\beta 1$ contributes to breast cancer cell migration, invasion, and metastasis (22-24). Whether HRG- $\beta 1$ can induce glycolysis, and whether HRG- $\beta 1$ induced glycolysis contributes to cell migration, is unknown. To explore HRG- $\beta 1$ 's ability to induce glycolysis, MCF7 cells were used because they express ErbB2, ErbB3, and ErbB4 receptors (25).

In this study, we investigated the importance of FASN, ErbB2-mediated glycolysis in breast cancer cell migration. We report that FASN, ErbB2-high-expressing SK-BR-3 cells displayed higher levels of glycolysis and migration than FASN, ErbB2-low-expressing MCF7 cells. In addition, inhibition of FASN by cerulenin impaired glycolysis and migration in SK-BR-3 cells. Finally, we show that inhibition of glycolysis by $2-D G$ or OX reverses HRG- $\beta 1$-induced migration. These results indicate that breast cancer cells are dependent on FASN, ErbB2-mediated glycolysis for migration, suggesting that glycolysis is a novel factor influencing migration and that it plays an important role in breast cancer progression.

\section{Materials and methods}

Cell lines and cell cultures. Human breast cancer cell line MCF7 was obtained from the Cell Bank of Chinese Academy of Sciences, Shanghai, China. SK-BR-3 was a gift from Professor Shengyong Yang of Sichuan University. MCF7 cells were cultured in DMEM/F12 (Hyclone) with $10 \%$ fetal bovine serum (FBS, Biological Industries) and penicillin/ streptomycin (PS, Hyclone). SK-BR-3 cells were maintained in DMEM (Hyclone) supplemented with 10\% FBS and PS. Cells were cultured at $37^{\circ} \mathrm{C}$ in a $5 \% \mathrm{CO}_{2}$ incubator.

Cell treatments with reagents. SK-BR-3 cells ( $1 \times 10^{6}$ cells/well) were plated in 6-well plates for $24 \mathrm{~h}$ and DMSO or cerulenin (10 $\mu \mathrm{g} / \mathrm{ml}$, Cayman) was added into the medium. Twenty-four hours after treatment, media was collected for glucose uptake and lactate production assays and cells were trypsinized for Transwell and western blot assays. MCF7 cells $\left(8 \times 10^{5}\right.$ cells/ well) were plated in 6-well plates for $24 \mathrm{~h}$. The medium was changed to $0.5 \%$ FBS medium and cells were starved for 24 h. Cells were treated with vehicle, heregulin- $\beta 1$ (HRG- $\beta 1$, $100 \mathrm{ng} / \mathrm{ml}$, PeproTech), HRG- $\beta 1$ plus cerulenin $(10 \mu \mathrm{g} / \mathrm{ml})$, 2-deoxyglucose (2-DG, $0.5 \mathrm{mM}$, Sigma) or oxamate (OX,
$50 \mathrm{mM}$, Sigma). $24 \mathrm{~h}$ after treatment, media was collected for glucose uptake and lactate production assays and cells were trypsinized for Transwell and western blot assays.

Glucose uptake and lactate production assays. Glucose uptake was measured using Glucose Oxidase Assay kit (Applygen, China) and results were normalized to the total cellular protein amounts. Lactate production in the medium was performed by using Lactate Assay kit (Nanjing Jiancheng Bioengineering Institute, China) according to the manufacturer's protocol. Results were normalized to the total cellular protein amounts.

Western blot assay. Cells were harvested and lysed in a buffer containing $50 \mathrm{mM}$ Tris- $\mathrm{HCl}, \mathrm{pH} 7.5,150 \mathrm{mM} \mathrm{NaCl}$, $2 \mathrm{mM}$ EDTA, $1 \%$ Triton, $1 \mathrm{mM}$ PMSF and Protease Inhibitor Cocktail (Sigma) for $20 \mathrm{~min}$ on ice. Lysates were cleared by centrifugation at $15,000 \mathrm{~g}$ at $4^{\circ} \mathrm{C}$ for $20 \mathrm{~min}$. Supernatants were collected and protein concentrations were determined by Bradford assay (Bio-Rad). Proteins were subjected to electrophoresis on SDS-polyacrylamide gels and were then transferred to PVDF membranes (Bio-Rad). Membranes were blocked in Tris buffered saline (TBS) (pH 7.4) with 5\% non-fat dry milk and probed with primary antibodies as indicated in the figure legends. The following antibodies were used: FASN (sc-55580, Santa Cruz), ErbB2 (2156, Cell Signaling Technology), LDHA (3582, Cell Signaling Technology), $\beta$-actin (A2228, Sigma), glyceraldehyde-3-phosphate dehydrogenase (GAPDH) (AG019, Beyotime, China). Immunoreactive bands were visualized by horseradish peroxidase (HRP)-conjugated secondary antibodies (Bio-Rad) using ECL Western Blotting Substrate (Pierce). The fold expression of protein was quantified using Image Lab software. Protein expression in control group was set as 1.0.

Cell transfection. pcDNA3.0 was purchased from Hangzhou Baosai Biotechnology, China and pcDNA3.0/ErbB2 was purchased from Addgene. Transient transfection was performed using the Lipofectamine 2000 transfection reagent (Invitrogen) according to the manufacturer's protocol. Forty-eight hours after transfection, cells were trypsinized for Transwell and western blot assays, and culture media was collected for glucose uptake and lactate production assays.

Wound healing assay. MCF7 and SK-BR-3 cells were plated in 6-well plates and allowed to grow to confluence. Wounds were made using a $10 \mu \mathrm{l}$ pipette tip and indicated reagents were added to the cells. Wound closure was monitored over a 24 or $48 \mathrm{~h}$ period and photographs were taken at 0,24 or $48 \mathrm{~h}$ after wounding. Wound width at 0,24 or $48 \mathrm{~h}$ was measured and the results were expressed as wound closure $(\%)=($ width at $0 \mathrm{~h}$ - width at $\mathrm{x} \mathrm{h}) /$ width at $0 \mathrm{~h} \times 100 \%(\mathrm{x}=24$ or 48$)$.

Transwell migration assay. Transwell migration assay was performed using Falcon cell culture inserts (Corning). Cells were trypsinized and suspended in medium with $0.5 \% \mathrm{FBS}$. MCF7 cells $\left(3 \times 10^{5}\right)$ or SK-BR-3 cells $\left(1 \times 10^{5}\right)$ were seeded in the top of the inserts and placed in the wells containing medium with $10 \% \mathrm{FBS}$. After $48 \mathrm{~h}$ incubation at $37^{\circ} \mathrm{C}$, the inserts were washed with PBS twice and cells on the upper surface of the inserts were gently removed with a cotton swab. 

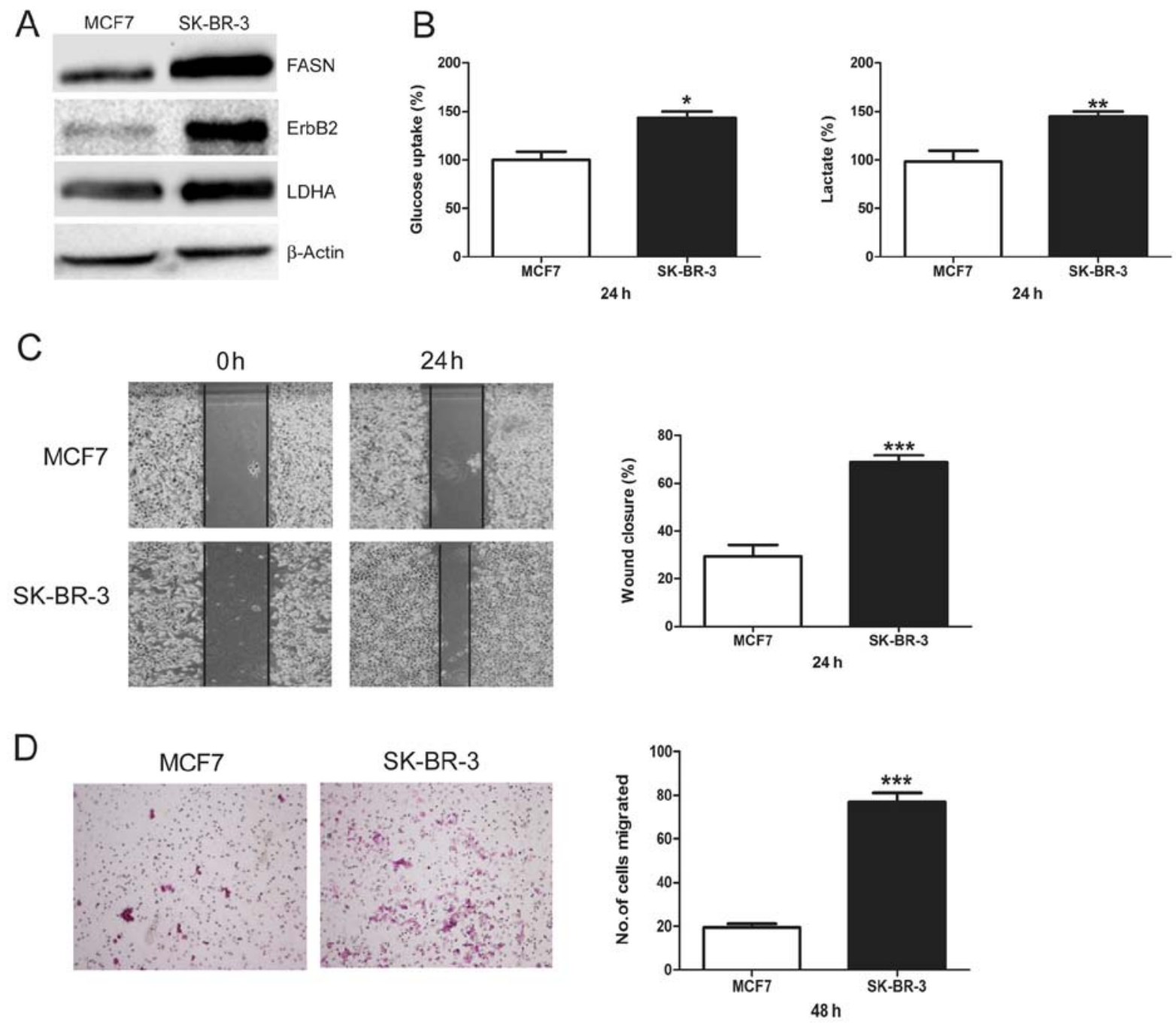

Figure 1. FASN, ErbB2-overexpressing SK-BR-3 cells possess a higher level of glycolysis and migratory potential than FASN, ErbB2-low-expressing MCF7 cells. (A) FASN, ErbB2 and LDHA protein expression in MCF7 and SK-BR-3 cells were detected by western blotting. $\beta$-actin was used as a loading control. (B) Glucose uptake and lactate production were compared between MCF7 and SK-BR-3 cells. Data are shown in percentage relative to MCF7 cells. (C) Confluent monolayers of MCF7 and SK-BR-3 cells were wounded using a pipette tip. Wound closure was monitored by microscopy and representative photomicrographs (x40 magnification) are shown (left). Wound width was measured at 0 and $24 \mathrm{~h}$ and plotted as percentage of wound closure (right). (D) Transwell migration assay. MCF7 and SK-BR-3 cells were plated into inserts and after $48 \mathrm{~h}$ of incubation, migrated cell numbers were counted under a microscope in five random fields per insert. Columns, mean of three independent experiments; bars, $\mathrm{SE}$; ${ }^{*} \mathrm{P}<0.05,{ }^{* *} \mathrm{P}<0.01,{ }^{* * * *} \mathrm{P}<0.001$.

The inserts were fixed with $10 \%$ methanol, rinsed with ultrapure water, and stained with $0.1 \%$ crystal violet for $20 \mathrm{~min}$. Cells that migrated onto the lower surface were counted under a microscope in five random fields.

Statistical analysis. All experiments were repeated three times. Statistical analysis was performed using GraphPad Prism version 5.0. The data were expressed as means \pm SE. The difference between groups was detected using Student's t-test. A value of $\mathrm{P}<0.05$ was considered to indicate a statistically significant difference.

\section{Results}

FASN, ErbB2-high-expressing SK-BR-3 cells show increased glycolysis and migration versus FASN, ErbB2-low-expressing MCF 7 cells. First, we demonstrated that MCF7 cells displayed low levels of FASN and ErbB2 and SK-BR-3 cells had high levels of FASN and ErbB2 (Fig. 1A). Then, we compared LDHA protein levels between these two cell lines. We found that SK-BR-3 cells showed much higher LDHA protein levels than MCF7 cells (Fig. 1A). Next, glucose uptake and lactate production, which are two important markers of glycolysis, were measured and compared between MCF7 and SK-BR-3 cells. SK-BR-3 cells showed a significantly higher glucose uptake and lactate production than MCF7 cells (Fig. 1B). Finally, we compared the migratory potential between MCF7 and SK-BR-3 cells using wound healing assay and Transwell assay. Compared to MCF7 cells, SK-BR-3 cells showed a higher percentage of wound closure (Fig. 1C) and migrated cell numbers (Fig. 1D), indicating that SK-BR-3 cells had a greater basal migration capacity than MCF7 cells. These results suggest a consistent relationship between FASN, ErbB2, glycolysis, and cell migration, indicating that increased glycolysis predicts higher migratory potential in breast cancer cells. 

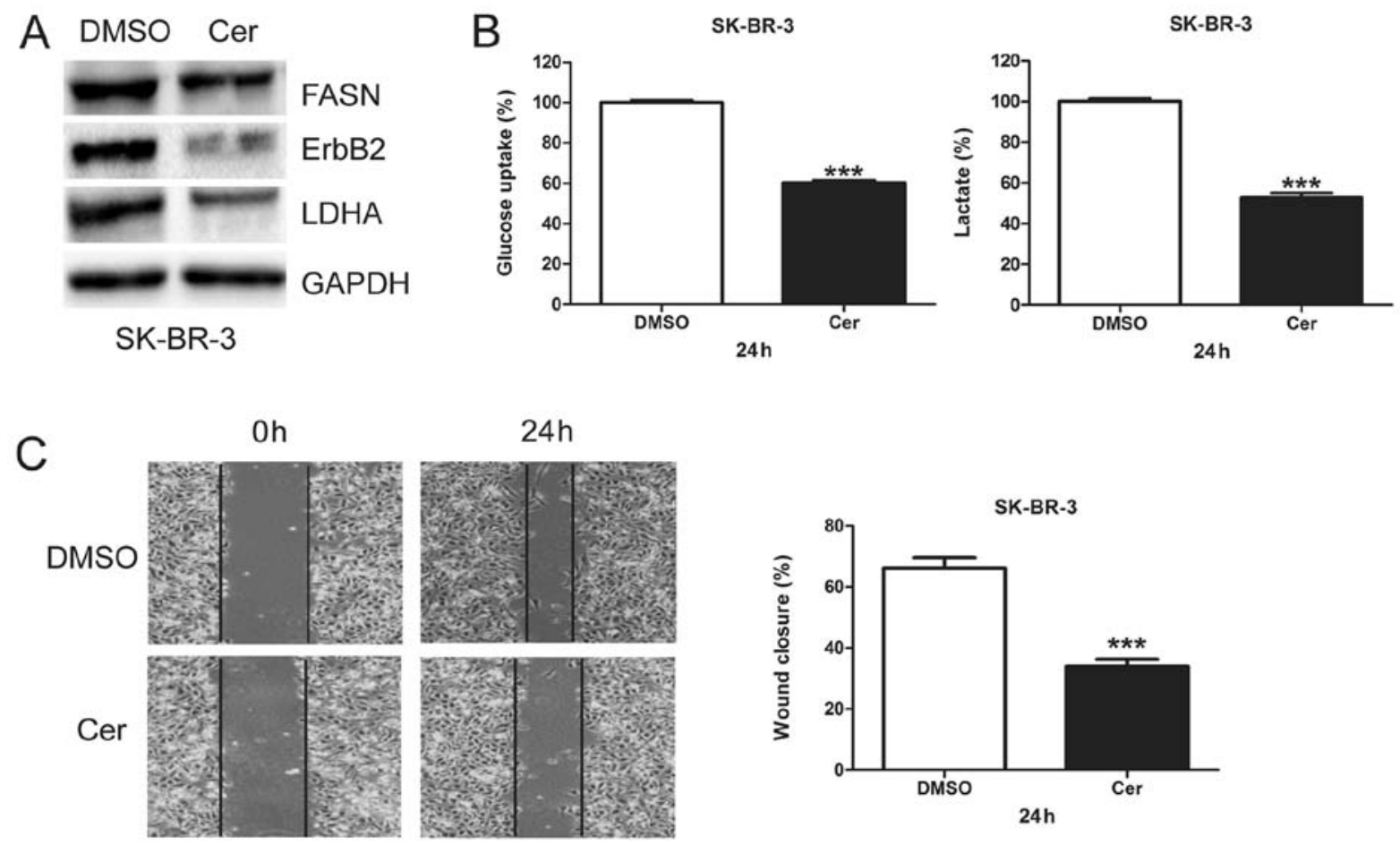

\section{SK-BR-3}

D

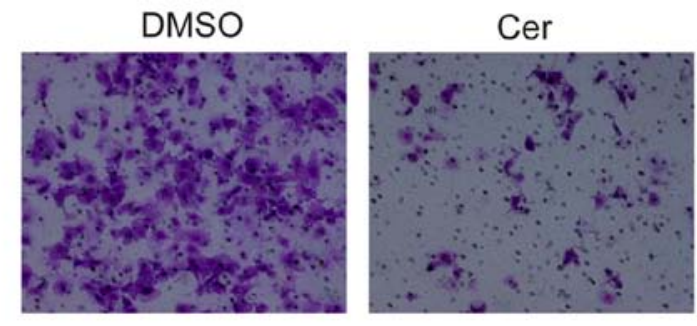

SK-BR-3

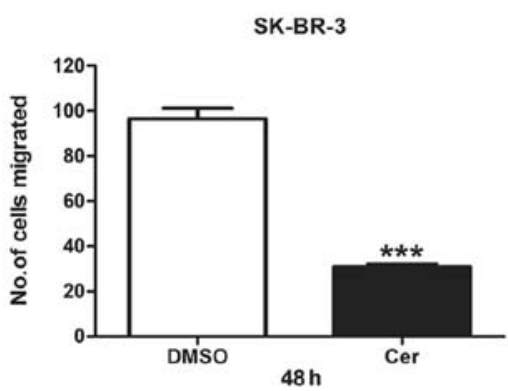

Figure 2. Cerulenin inhibits glycolysis and migration in SK-BR-3 cells. (A) SK-BR-3 cells were treated with DMSO or cerulenin (Cer, $10 \mu \mathrm{g} / \mathrm{ml})$ for $24 \mathrm{~h}$ and FASN, ErbB2, LDHA protein levels were detected by western blotting. GAPDH was used as a loading control. (B) SK-BR-3 cells were treated with DMSO or Cer $(10 \mu \mathrm{g} / \mathrm{ml})$ for $24 \mathrm{~h}$ and glucose uptake and lactate production were measured in the medium. Data are shown in percentage relative to DMSO-treated cells. (C) Confluent monolayers of SK-BR-3 cells were wounded using a pipette tip and DMSO or Cer (10 $\mu \mathrm{g} / \mathrm{ml})$ were added into the medium. Wound closure was monitored by microscopy and representative photomicrographs (x40 magnification) are shown (left). Wound width was measured at 0 and $24 \mathrm{~h}$ and plotted as percentage of wound closure (right). (D) SK-BR-3 cells were treated with DMSO or Cer (10 $\mu \mathrm{g} / \mathrm{ml})$ for $24 \mathrm{~h}$. Then cells were plated into inserts and after $48 \mathrm{~h}$ of incubation, migrated cell numbers were counted under a microscope in five random fields per insert. Columns, mean of three independent experiments; bars, $\mathrm{SE} ;{ }^{* * * *} \mathrm{P}<0.001$.

Cerulenin decreases glycolysis and migration in $S K-B R-3$ cells. To investigate the role of FASN in glycolysis and cell migration, we treated SK-BR-3 cells with cerulenin, a specific inhibitor of FASN, and measured its effects on ErbB2 and LDHA protein levels, glycolysis, and cell migration. When FASN protein levels were inhibited by cerulenin, both ErbB2 and LDHA proteins levels were downregulated (Fig. 2A). Cerulenin treatment in SK-BR-3 cells also significantly decreased glucose uptake and lactate production (Fig. 2B), indicating that inhibition of FASN by cerulenin inhibits glycolysis. We further found that cerulenin significantly decreased migratory capacity of SK-BR-3 cells (Fig. 2C and D). These results suggest FASN may play an important role in glycolysis and migration of breast cancer cells.

Overexpression of ErbB2 promotes glycolysis and cell migration. To investigate the role of ErbB2-mediated glycolysis in cell migration, we transiently transfected ErbB2 plasmid into MCF7 cells and then detected FASN and LDHA protein levels. We found that overexpression of ErbB2 upregulates FASN and LDHA protein levels (Fig. 3A). Moreover, we found that acute overexpression of ErbB2 increased both glucose uptake and lactate production (Fig. 3B), indicating increased glycolysis. Then, the role of ErbB2-mediated glycolysis in cell migration was investigated, and we found that overexpression of ErbB2 greatly enhanced cell migration (Fig. 3C). These results suggest ErbB2 plays an important role in glycolysis and migration of breast cancer cells.

Cerulenin reverses $H R G$ - $\beta 1$-induced glycolysis and migration in MCF7 cells. We treated MCF7 cells with HRG- $\beta 1$ and detected FASN, ErbB2, and LDHA protein levels. We found that HRG- $\beta 1$ upregulated FASN, ErbB2 and LDHA protein levels whereas cerulenin reversed such upregulation (Fig. 4A). 

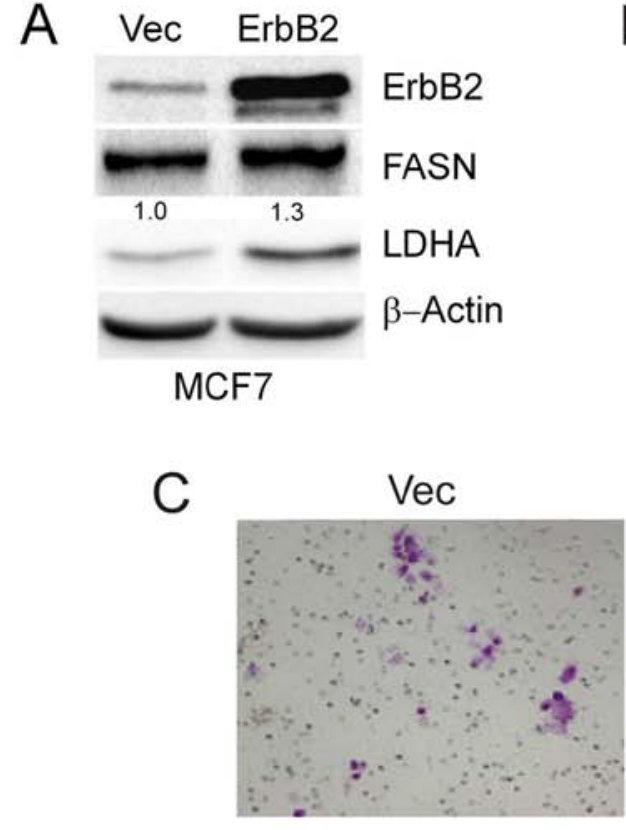
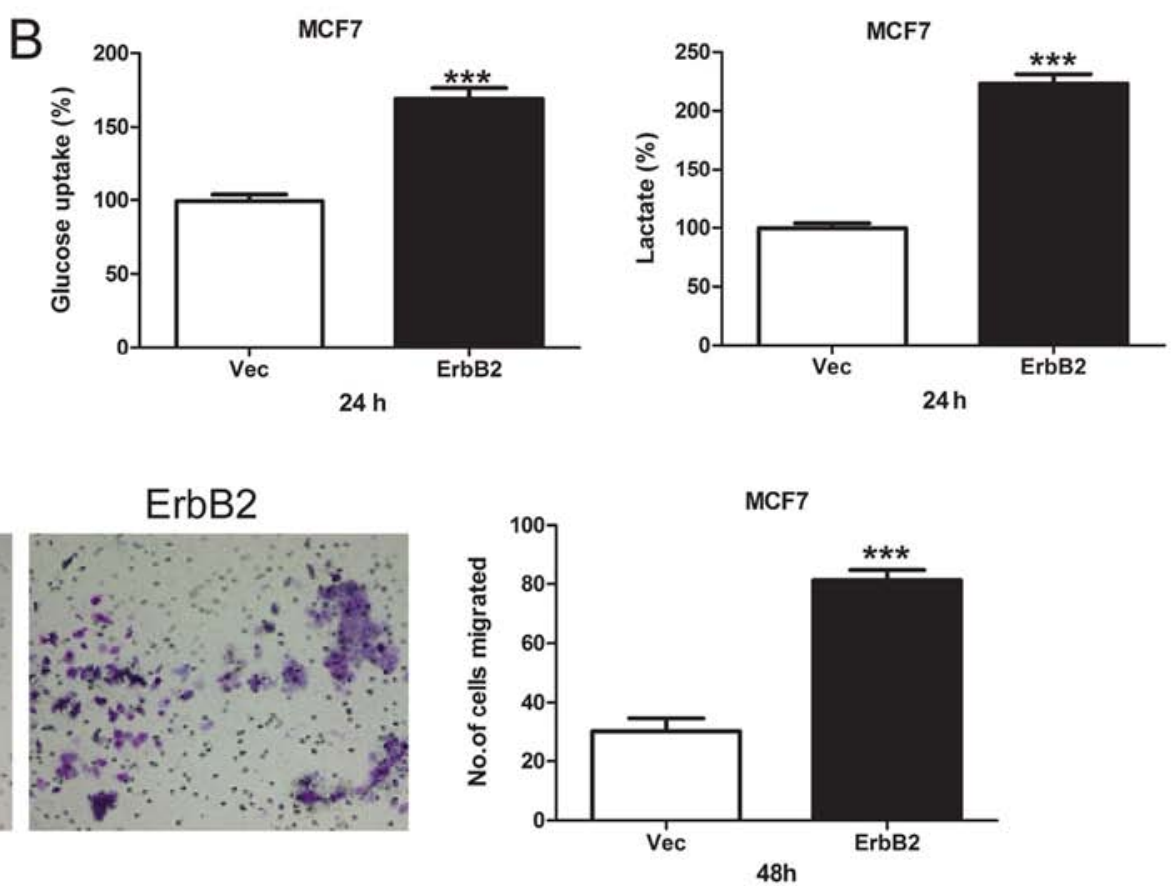

Figure 3. Overexpression of ErbB2 promotes glycolysis and cell migration. (A) Western blot analysis of ErbB2, FASN and LDHA protein levels in MCF7 cells transiently transfected with pcDNA3.0 (Vec) or pcDNA3.0/ErbB2 (ErbB2) plasmid. The $\beta$-actin protein served as a loading control. The fold expression of FASN was quantified using the protein level in Vec-transfected MCF7 cells as 1.0. (B) MCF7 cells were transfected with Vec or ErbB2 plasmid and $48 \mathrm{~h}$ after transfection, cells were transferred to 24 -well plates for glucose uptake and lactate production assay. Data are shown in percentage relative to Vec-transfected MCF7 cells. (C) MCF7 cells were transfected with Vec or ErbB2 plasmid and $48 \mathrm{~h}$ after transfection, cells were plated into inserts for Transwell migration assay. After $48 \mathrm{~h}$ of incubation, migrated cell numbers were counted under a microscope in five random fields per insert. Columns, mean of three independent experiments; bars, $\mathrm{SE} ;{ }^{* * * *} \mathrm{P}<0.001$.

We further found that HRG- $\beta 1$ induced both glucose uptake and lactate production with cerulenin reversing such an induction in MCF7 cells (Fig. 4B). Moreover, HRG- $\beta 1$ treatment in MCF7 cells greatly increased wound closure and number of migrated cells, while cerulenin overcame such increases (Fig. 4C and D). This result suggests that FASN plays an important role in HRG- $\beta 1$-indcued glycolysis and migration.

2-DG or OX reverses HRG- $\beta 1$-induced glycolysis and migration in MCF7 cells. In order to further demonstrate that glycolysis is required for cell migration, we treated MCF7 cells with HRG- $\beta 1$ and detected LDHA protein levels. We found that HRG- $\beta 1$ greatly upregulated LDHA protein levels while both 2-DG and OX reversed the upregulation (Fig. 5A). Moreover, both 2-DG and OX reversed HRG- $\beta 1$-induced glycolysis (Fig. 5B) and cell migration (Fig. 5C and D). These results suggest that $\mathrm{HRG}-\beta 1$-induced glycolysis promotes cell migration whereas glycolysis inhibition by 2-DG or OX reverses such effects.

\section{Discussion}

Thus far, there has been no evidence reported to directly link glycolysis to cell migration. Here, for the first time, we have shown that FASN, ErbB2-mediated glycolysis is required for breast cancer cell migration. We found that FASN, ErbB2overexpressing SK-BR-3 cells displayed higher LDHA protein levels, a higher level of glycolysis, and a greater migratory capacity when compared to FASN, ErbB2-low-expressing
MCF7 cells. Inhibition of FASN by cerulenin decreased ErbB2 and LDHA protein levels, as well as glycolysis and migration in SK-BR-3 cells. Activation of ErbB2 by overexpression, or by HRG- $\beta 1$, promotes glycolysis and cell migration while inhibition of glycolysis by 2-DG or OX reverses such induction. These results demonstrated that glycolysis is directly linked to breast cancer cell migration.

Lactate is the end product of glycolysis and a number of studies have shown that it plays important roles in the malignant development of tumor, such as cell migration, invasion and metastasis (26-29). However, all in vitro studies have been performed by treating cancer cells with exogenous lactate. To our knowledge, this is the first report showing that endogenous lactate directly induced by glycolysis promotes breast cancer cell migration.

ErbB2 and FASN are reciprocally regulated in breast cancer cells. On the one hand, overexpression of ErbB2 upregulates FASN expression, both at the transcriptional level and at the translational level $(10,30)$. Activation of ErbB2 by HRG- $\beta 1$ induces tyrosine phosphorylation of FASN and upregulation of FASN activity, leading to increased cell invasion (31). On the other hand, overexpression of FASN activates ErbB2 and induces an invasive breast cancer-like phenotype (18) and FASN regulates ErbB2 expression at the transcriptional level via PEA3 (32). Our results showed that both ErbB2 and FASN protein levels are directly consistent with LDHA protein level, glycolysis, and migration, while inhibition of FASN also decreased ErbB2 and LDHA protein levels, glycolysis, and migration. Our previous study (19) showed that ErbB2 upregu- 

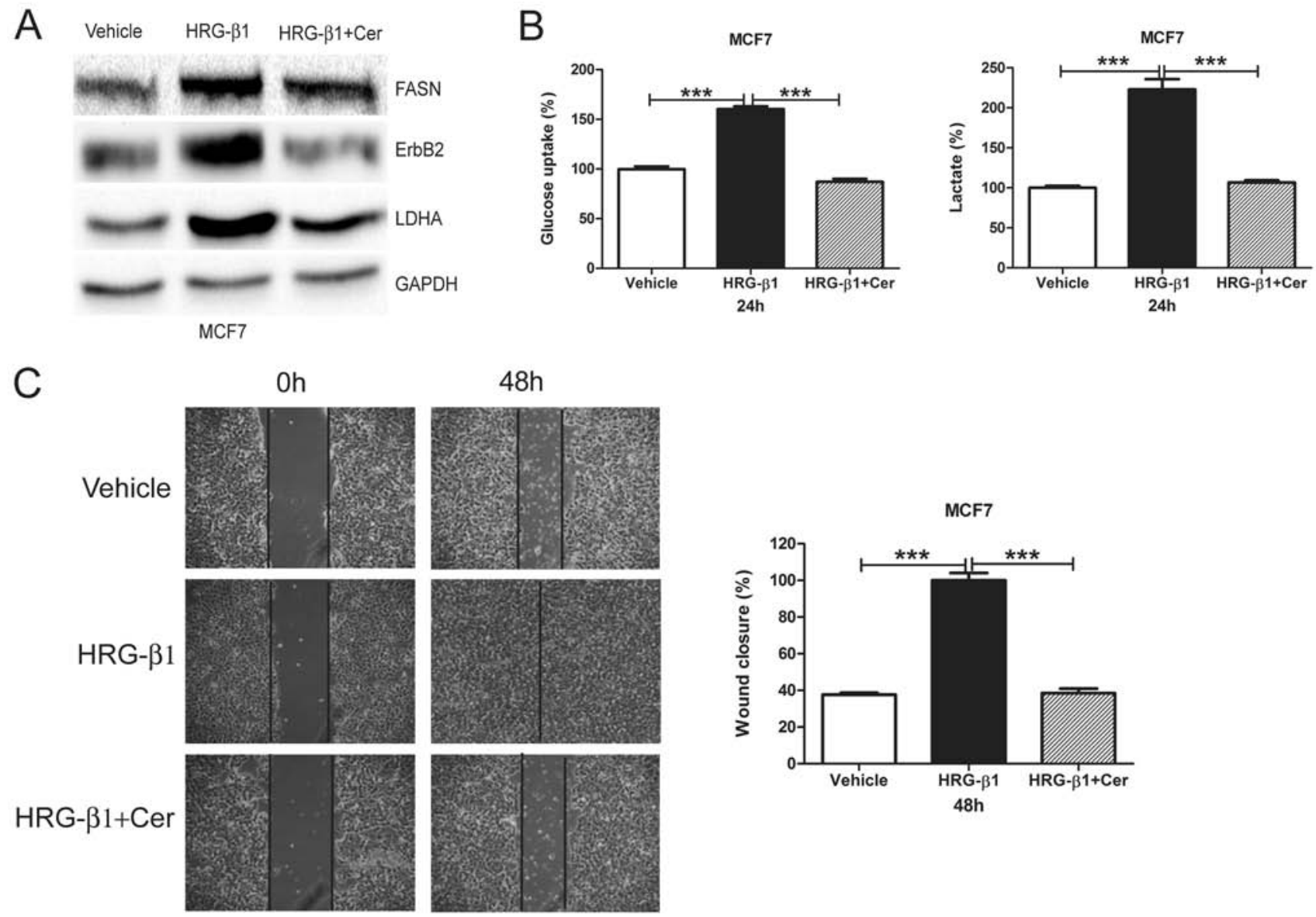

MCF7
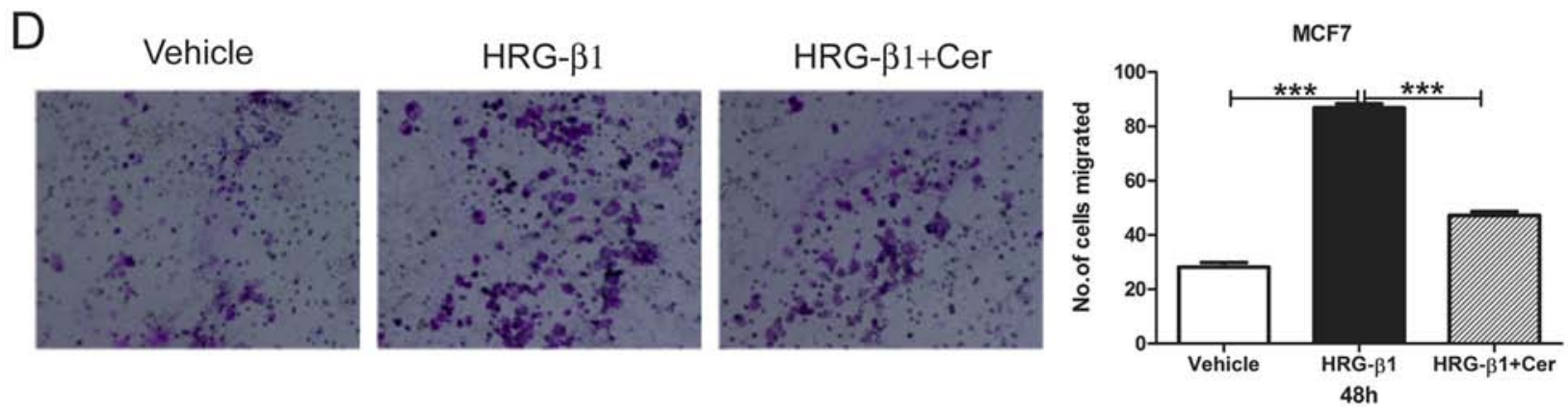

Figure 4. Cerulenin reverses HRG- $\beta 1$-induced glycolysis and cell migration. (A) MCF7 cells were treated with Vehicle, HRG- $\beta 1$ (100 ng/ml), HRG- $\beta 1$ $(100 \mathrm{ng} / \mathrm{ml})$ plus Cer $(10 \mu \mathrm{g} / \mathrm{ml})$ for $24 \mathrm{~h}$. Cells were collected and western blot analysis of ErbB2, FASN and LDHA protein levels was performed. GAPDH served as a loading control. (B) MCF7 cells were treated with Vehicle, HRG- $\beta 1(100 \mathrm{ng} / \mathrm{ml})$, HRG- $\beta 1(100 \mathrm{ng} / \mathrm{ml}) \mathrm{plus}$ Cer $(10 \mu \mathrm{g} / \mathrm{ml})$ for $24 \mathrm{~h}$. Cell media were collected for glucose uptake and lactate production assay. Data are shown in percentage relative to Vehicle-treated MCF7 cells. (C) Confluent monolayers of MCF7 cells were wounded using a pipette tip and were treated with Vehicle, HRG- $\beta 1$ (100 ng/ml), HRG- $\beta 1$ (100 ng/ml) plus Cer (10 $\mu \mathrm{g} / \mathrm{ml})$ for $48 \mathrm{~h}$. Wound closure was monitored by microscopy and representative photomicrographs (x40 magnification) are shown (left). Wound width was measured at 0 and $48 \mathrm{~h}$ and plotted as percentage of wound closure (right). (D) MCF7 cells were treated with Vehicle, HRG- $\beta 1$ (100 ng/ml), HRG- $\beta 1$ (100 ng/ml) plus Cer (10 $\mu \mathrm{g} / \mathrm{ml})$ for $24 \mathrm{~h}$. Then, cells were plated into inserts for Transwell migration assay. After $48 \mathrm{~h}$ of incubation, migrated cell numbers were counted under a microscope in five random fields per insert. Columns, mean of three independent experiments; bars, $\mathrm{SE}$; ${ }^{* * *} \mathrm{P}<0.001$.

lates LDHA at the transcriptional level. However, whether FASN directly regulates LDHA, or indirectly regulates it via ErbB2, deserves further investigation.

There are several possible mechanisms by which HRG- $\beta 1$ drives breast cancer cell migration and invasion: these include upregulation of tumor necrosis factor receptor (TNFR) superfamily member Fn14 expression (25), induction of EMT $(22,23)$, and upregulation of GPR30 expression $(24,33)$. We have found a novel mechanism whereby HRG- $\beta 1$ can induce glycolysis and drive migration in MCF7 cells. Phosphatidylinositol 3-kinase (PI3K) and mitogen-activated protein kinase (MAPK) are two important pathways downstream of ErbB2 signaling induced by HRG- $\beta 1$ (34). PI3K pathway plays a dominant role in cellular transformation in ErbB2/ErbB3 coreceptor-mediated heregulin signaling (34) and AKT stimulates aerobic glycolysis in cancer cells (35). Therefore, we speculate that activation of PI3K/AKT pathway by HRG- $\beta 1$ promotes glycolysis resulting in enhanced cell migration. 


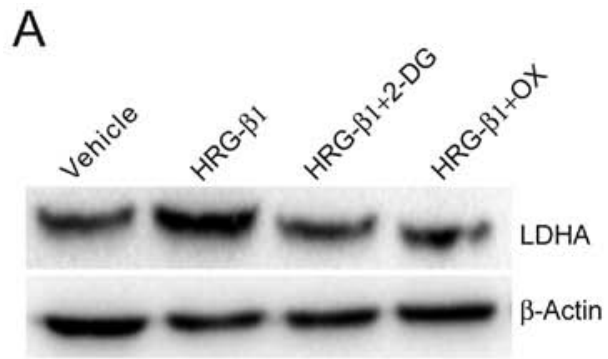

B
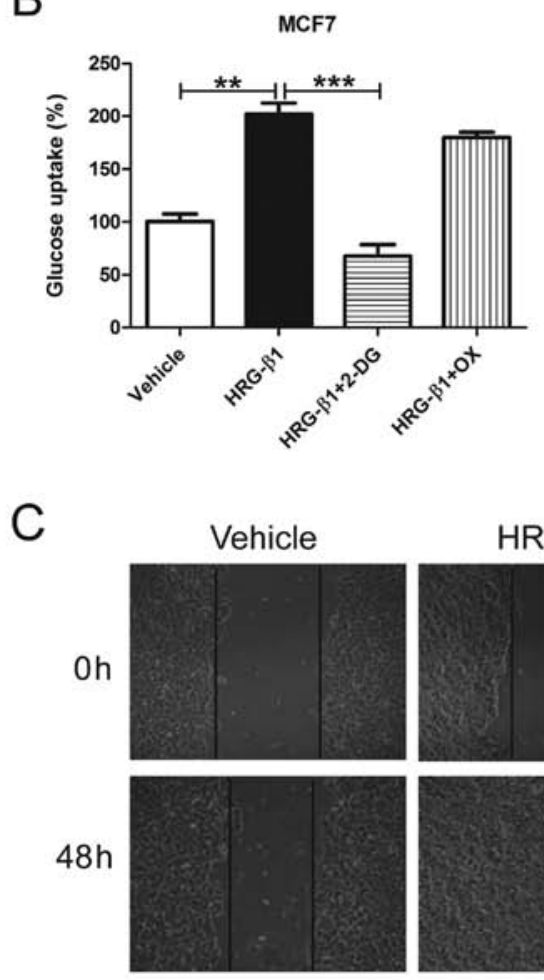

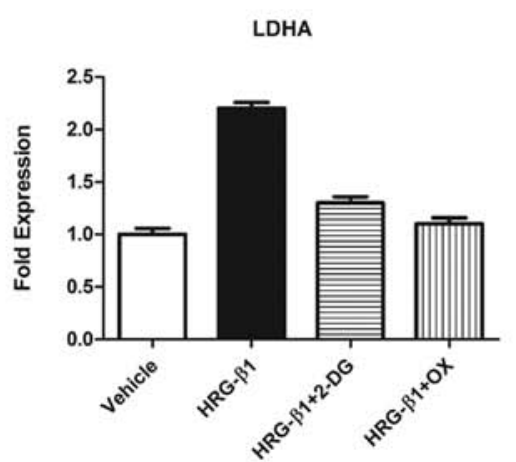

MCF7
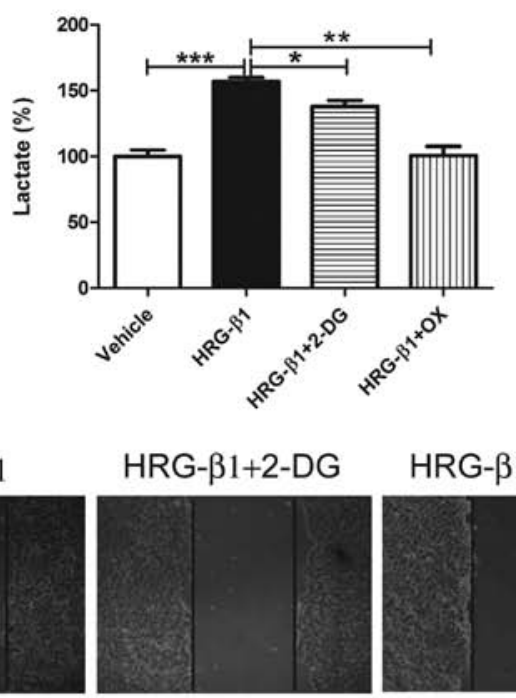

HRG- $\beta 1+O X$
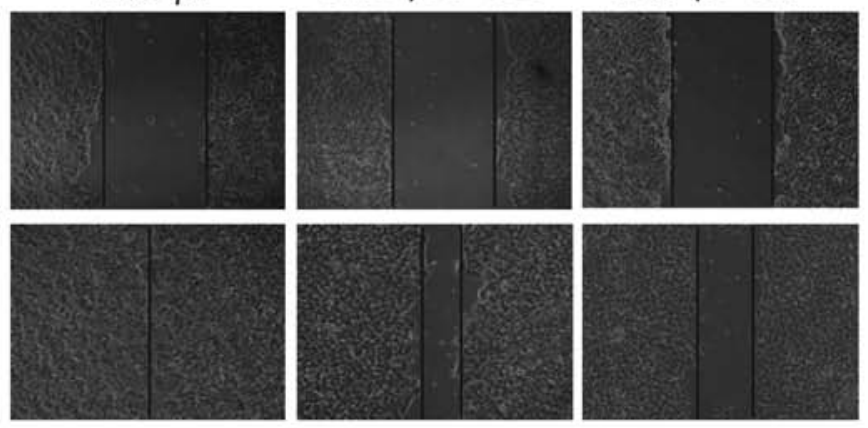

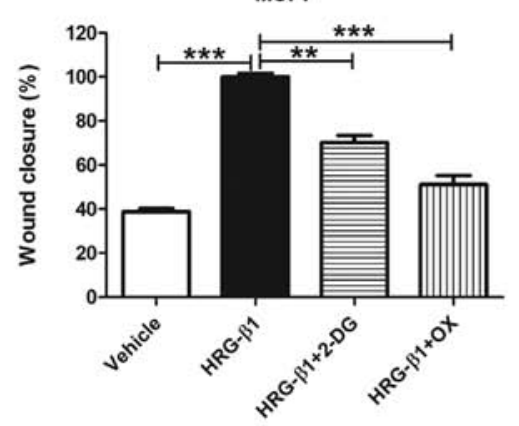

Figure 5. 2-DG and OX reverses HRG- $\beta 1$-induced glycolysis and cell migration. (A) MCF7 cells were treated with Vehicle, HRG- $\beta 1$ (100 ng/ml), HRG- $\beta 1$ $(100 \mathrm{ng} / \mathrm{ml})$ plus 2-DG $(0.5 \mathrm{mM})$ or OX $(50 \mathrm{mM})$ for $24 \mathrm{~h}$. Cells were collected and western blot analysis of LDHA protein levels was performed. $\beta$-actin served as a loading control (left). The fold expression of LDHA was quantified using the protein level treated with vehicle as 1.0 (right). (B) MCF7 cells were treated with Vehicle, HRG- $\beta 1(100 \mathrm{ng} / \mathrm{ml}), \mathrm{HRG}-\beta 1(100 \mathrm{ng} / \mathrm{ml})$ plus 2-DG $(0.5 \mathrm{mM})$ or OX $(50 \mathrm{mM})$ for $24 \mathrm{~h}$. Cell media were collected for glucose uptake and lactate production assay. Data are shown in percentage relative to Vehicle-treated MCF7 cells. (C) Confluent monolayers of MCF7 cells were wounded using a pipette tip and were treated with Vehicle, HRG- $\beta 1(100 \mathrm{ng} / \mathrm{ml})$, HRG- $\beta 1(100 \mathrm{ng} / \mathrm{ml})$ plus 2-DG $(0.5 \mathrm{mM})$ or OX $(50 \mathrm{mM})$ for 48 h. Wound closure was monitored by microscopy and representative photomicrographs (x40 magnification) are shown. Wound width was measured at 0 and $48 \mathrm{~h}$ and plotted as percentage of wound closure. Columns, mean of three independent experiments; bars, $\mathrm{SE} .{ }^{*} \mathrm{P}<0.05,{ }^{* * *} \mathrm{P}<0.01,{ }^{* * *} \mathrm{P}<0.001$.

In summary, to the best of our knowledge, for the first time, we report that FASN, ErbB2-mediated glycolysis is required for breast cancer cell migration. Inhibition of FASN by cerulenin, or inhibition of glycolysis by 2-DG or OX, reverses HRG- $\beta 1$ - induced glycolysis and migration. This study provides direct evidence in support of a causal relationship between glycolysis and breast cancer cell migration. These novel findings prompt us to further investigate the role of glycolysis in invasion and 
metastasis of breast cancer, which is important for elucidating the molecular mechanism of breast cancer metastasis.

\section{Acknowledgements}

The authors acknowledge financial support for the projects supported by National Natural Sciences Foundation of China (81272907, J1103604) and the Project 2012-1707-7-7 sponsored by the Scientific Research Foundation for the Returned Overseas Chinese Scholars, State Education Ministry.

\section{References}

1. Torre LA, Bray F, Siegel RL, Ferlay J, Lortet-Tieulent J and Jemal A: Global cancer statistics, 2012. CA Cancer J Clin 65 87-108, 2015.

2. Weigelt B, Peterse JL and van 't Veer LJ: Breast cancer metastasis: Markers and models. Nat Rev Cancer 5: 591-602, 2005.

3. Scully OJ, Bay BH, Yip G and Yu Y: Breast cancer metastasis. Cancer Genomics Proteomics 9: 311-320, 2012.

4. Hanahan D and Weinberg RA: Hallmarks of cancer: The next generation. Cell 144: 646-674, 2011

5. Hsu PP and Sabatini DM: Cancer cell metabolism: Warburg and beyond. Cell 134: 703-707, 2008.

6. Kim JW and Dang CV: Cancer's molecular sweet tooth and the Warburg effect. Cancer Res 66: 8927-8930, 2006.

7. Kroemer G and Pouyssegur J: Tumor cell metabolism: Cancer's Achilles' heel. Cancer Cell 13: 472-482, 2008.

8. Vander Heiden MG, Cantley LC and Thompson CB: Understanding the Warburg effect: The metabolic requirements of cell proliferation. Science 324: 1029-1033, 2009.

9. Warburg O: On respiratory impairment in cancer cells. Science 124: 269-270, 1956.

10. Kumar-Sinha C, Ignatoski KW, Lippman ME, Ethier SP and Chinnaiyan AM: Transcriptome analysis of HER2 reveals a molecular connection to fatty acid synthesis. Cancer Res 63 : 132-139, 2003

11. Menendez JA, Mehmi I, Atlas E, Colomer R and Lupu R: Novel signaling molecules implicated in tumor-associated fatty acid synthase-dependent breast cancer cell proliferation and survival: Role of exogenous dietary fatty acids, p53-p21WAF1/CIP1, ERK1/2 MAPK, p27KIP1, BRCA1, and NF-kappaB. Int J Oncol 24: 591-608, 2004.

12. Slamon DJ, Godolphin W, Jones LA, Holt JA, Wong SG, Keith DE, Levin WJ, Stuart SG, Udove J, Ullrich A, et al: Studies of the HER-2/neu proto-oncogene in human breast and ovarian cancer. Science 244: 707-712, 1989.

13. Guy CT, Webster MA, Schaller M, Parsons TJ, Cardiff RD and Muller WJ: Expression of the neu protooncogene in the mammary epithelium of transgenic mice induces metastatic disease. Proc Natl Acad Sci USA 89: 10578-10582, 1992.

14. Johnson E, Seachrist DD, DeLeon-Rodriguez CM, Lozada KL, Miedler J, Abdul-Karim FW and Keri RA: HER2/ErbB2-induced breast cancer cell migration and invasion require p120 catenin activation of Rac1 and Cdc42. J Biol Chem 285: 29491-29501, 2010.

15. Li J, Dong L, Wei D, Wang X, Zhang S and Li H: Fatty acid synthase mediates the epithelial-mesenchymal transition of breast cancer cells. Int J Biol Sci 10: 171-180, 2014.

16. Tan M, Li P, Klos KS, Lu J, Lan KH, Nagata Y, Fang D, Jing $\mathrm{T}$ and $\mathrm{Yu} \mathrm{D}$ : ErbB2 promotes Src synthesis and stability: Novel mechanisms of Src activation that confer breast cancer metastasis. Cancer Res 65: 1858-1867, 2005.

17. Tan M, Yao J and Yu D: Overexpression of the c-erbB-2 gene enhanced intrinsic metastasis potential in human breast cancer cells without increasing their transformation abilities. Cancer Res 57: 1199-1205, 1997.

18. Vazquez-Martin A, Colomer R, Brunet J, Lupu R and Menendez JA: Overexpression of fatty acid synthase gene activates HER1/HER2 tyrosine kinase receptors in human breast epithelial cells. Cell Prolif 41: 59-85, 2008.
19. Zhao YH, Zhou M, Liu H, Ding Y, Khong HT, Yu D, Fodstad O and Tan M: Upregulation of lactate dehydrogenase A by ErbB2 through heat shock factor 1 promotes breast cancer cell glycolysis and growth. Oncogene 28: 3689-3701, 2009.

20. Montero JC, Rodríguez-Barrueco R, Ocaña A, Díaz-Rodríguez E, Esparís-Ogando A and Pandiella A: Neuregulins and cancer. Clin Cancer Res 14: 3237-3241, 2008.

21. Agus DB, Akita RW, Fox WD, Lewis GD, Higgins B, Pisacane PI, Lofgren JA, Tindell C, Evans DP, Maiese K, et al: Targeting ligand-activated ErbB2 signaling inhibits breast and prostate tumor growth. Cancer Cell 2: 127-137, 2002.

22. Cheng L, Zha Z, Lang B, Liu J and Yao X: Heregulin-beta1 promotes metastasis of breast cancer cell line SKBR3 through upregulation of Snail and induction of epithelial-mesenchymal transition. Cancer Lett 280: 50-60, 2009.

23. Kim J, Jeong H, Lee Y, Kim C, Kim H and Kim A: HRG- $\beta 1$ driven ErbB3 signaling induces epithelial-mesenchymal transition in breast cancer cells. BMC Cancer 13: 383, 2013.

24. Ruan SQ, Wang ZH, Wang SW, Fu ZX, Xu KL, Li DB and Zhang SZ: Heregulin- $\beta 1$-induced GPR 30 upregulation promotes the migration and invasion potential of $\mathrm{SkBr} 3$ breast cancer cells via ErbB2/ErbB3-MAPK/ERK pathway. Biochem Biophys Res Commun 420: 385-390, 2012.

25. Asrani K, Keri RA, Galisteo R, Brown SA, Morgan SJ, Ghosh A, Tran NL and Winkles JA: The HER2- and heregulin $\beta 1$ (HRG)-inducible TNFR superfamily member Fn14 promotes HRG-driven breast cancer cell migration, invasion, and MMP9 expression. Mol Cancer Res 11: 393-404, 2013

26. Bonuccelli G, Tsirigos A, Whitaker-Menezes D, Pavlides S, Pestell RG, Chiavarina B, Frank PG, Flomenberg N, Howell A, Martinez-Outschoorn UE, et al: Ketones and lactate 'fuel' tumor growth and metastasis: Evidence that epithelial cancer cells use oxidative mitochondrial metabolism. Cell Cycle 9: 3506-3514, 2010.

27. Goetze K, Walenta S, Ksiazkiewicz M, Kunz-Schughart LA and Mueller-Klieser W: Lactate enhances motility of tumor cells and inhibits monocyte migration and cytokine release. Int J Oncol 39: 453-463, 2011.

28. Hirschhaeuser F, Sattler UG and Mueller-Klieser W: Lactate: A metabolic key player in cancer. Cancer Res 71: 6921-6925, 2011.

29. Stern R, Shuster S, Neudecker BA and Formby B: Lactate stimulates fibroblast expression of hyaluronan and CD44: The Warburg effect revisited. Exp Cell Res 276: 24-31, 2002

30. Yoon S, Lee MY, Park SW, Moon JS, Koh YK, Ahn YH, Park BW and Kim KS: Up-regulation of acetyl-CoA carboxylase alpha and fatty acid synthase by human epidermal growth factor receptor 2 at the translational level in breast cancer cells. J Biol Chem 282: 26122-26131, 2007

31. Jin Q, Yuan LX, Boulbes D, Baek JM, Wang YN, GomezCabello D, Hawke DH, Yeung SC, Lee MH, Hortobagyi GN, et al: Fatty acid synthase phosphorylation: A novel therapeutic target in HER2-overexpressing breast cancer cells. Breast Cancer Res 12: R96, 2010.

32. Menendez JA, Vellon L, Mehmi I, Oza BP, Ropero S, Colomer R and Lupu R: Inhibition of fatty acid synthase (FAS) suppresses HER2/neu (erbB-2) oncogene overexpression in cancer cells. Proc Natl Acad Sci USA 101: 10715-10720, 2004.

33. Ruan SQ, Wang SW, Wang ZH and Zhang SZ: Regulation of HRG- $\beta 1$-induced proliferation, migration and invasion of MCF-7 cells by upregulation of GPR30 expression. Mol Med Rep 6: 131-138, 2012.

34. Vijapurkar U, Kim MS and Koland JG: Roles of mitogenactivated protein kinase and phosphoinositide 3'-kinase in ErbB2/ErbB3 coreceptor-mediated heregulin signaling. Exp Cell Res 284: 291-302, 2003.

35. Elstrom RL, Bauer DE, Buzzai M, Karnauskas R, Harris MH, Plas DR, Zhuang H, Cinalli RM, Alavi A, Rudin CM, et al: Akt stimulates aerobic glycolysis in cancer cells. Cancer Res 64: 3892-3899, 2004. 